\title{
Scalable and Dil-compatible optical clearance of the mammalian brain
}

\author{
Bing Hou ${ }^{1,2,3}$, Dan Zhang ${ }^{1+}$, Shan Zhao ${ }^{1+}$, Mengping Wei ${ }^{4}$, Zaifu Yang ${ }^{3}$, Shaoxia Wang ${ }^{3}$, Jiarui Wang ${ }^{3}$, \\ Xin Zhang ${ }^{1}$, Bing Liu ${ }^{1,2}$, Lingzhong Fan ${ }^{1}$, Yang Li $^{3}$, Zilong Qiu ${ }^{5}$, Chen Zhang ${ }^{4}$ and Tianzi Jiang ${ }^{1,2,6,78 *}$ \\ Brainnetome Center, Institute of Automation, Chinese Academy of Sciences, Beijing, China \\ ${ }^{2}$ National Laboratory of Pattern Recognition, Institute of Automation, Chinese Academy of Sciences, Beijing, China \\ ${ }^{3}$ Beijing Institute of Radiation Medicine, Beijing, China \\ ${ }^{4}$ State Key Laboratory of Biomembrane and Membrane Biotechnology, and PKU-IDG/McGovern Institute for Brain Research, School of Life Sciences, Peking \\ University, Beijing, China \\ ${ }^{5}$ Institute of Neuroscience, Shanghai Institutes for Biological Sciences, Shanghai, China \\ ${ }^{6}$ Queensland Brain Institute, The University of Queensland, Brisbane, QLD, Australia \\ Key Laboratory for Neurolnformation of Ministry of Education, School of Life Science and Technology, University of Electronic Science and Technology of China, \\ Chengdu, China \\ ${ }^{8}$ CAS Center for Excellence in Brain Science, Institute of Automation, Chinese Academy of Sciences, Beijing, China
}

\section{Edited by:}

Yun-Qing Li, The Fourth Military

Medical University, China

\section{Reviewed by:}

Carol Mason, Columbia University

Medical Center, USA

Hong-Wei Dong, University of

Southern California, USA

\section{*Correspondence:}

Tianzi Jiang, Brainnetome Center, Institute of Automation, Chinese Academy of Sciences, 95 Zhong Guan Cun East Road, Hai Dian District, Beijing 100190, China e-mail: jiangtz@n/pr.ia.ac.cn

${ }^{\dagger}$ These authors have contributed equally to this work.
Efficient optical clearance is fundamental for whole brain imaging. In particular, clearance of the brain without membrane damage is required for the imaging of lipophilic tracer-labeled neural tracts. Relying on an ascending gradient of fructose solutions, SeeDB can achieve sufficient transparency of the mouse brain while ensuring that the plasma membrane remains intact. However, it is challenging to extend this method to larger mammalian brains due to the extremely high viscosity of the saturated fructose solution. Here we report a SeeDB-derived optical clearing method, termed FRUIT, which utilizes a cocktail of fructose and urea. As demonstrated in the adult mouse brain, combination of these two highly water-soluble clearing agents exerts a synergistic effect on clearance. More importantly, the final FRUIT solution has low viscosity so as to produce transparency of the whole adult rabbit brain via arterial perfusion, which is impossible to achieve with a saturated fructose solution. In addition to good compatibility with enhanced yellow fluorescent protein, the cocktail also preserves the fluorescence of the lipophilic tracer Dil. This work provides a volume-independent optical clearing method which retains the advantages of SeeDB, particularly compatibility with lipophilic tracers.

Keywords: optical tissue clearing, SeeDB, Urea, tract tracing, whole brain imaging, CLARITY, CUBIC, 3DISCO

\section{INTRODUCTION}

Comprehensive depiction of structural layout and neuronal connectivity is indispensible for understanding how the mammalian brain functions. Due to high-throughput mechanical sectioning platforms and powerful automated three-dimensional imaging technologies, mesoscale atlases or connectomes of the entire mouse brain have been successfully reconstructed (Li et al., 2010; Oh et al., 2014). Despite these remarkable advances, the systemlevel relationship between brain structure and function often needs to be investigated, from a global perspective, in a whole brain rather than by reconstruction across preparations, in order to avoid loss of the detailed structure between sections (DeFelipe, 2010). However, sample opacity seriously limits the depth of imaging through the whole brain, particularly in adult animals. Recently, tissue optical clearing methods have opened a new avenue to extract cellular resolution information from unsectioned mammalian brains (Dodt et al., 2007; Hama et al., 2011; Becker et al., 2012; Erturk et al., 2012a; Chung et al., 2013; Ke et al., 2013; Kim et al., 2013; Kuwajima et al., 2013; Yushchenko and Schultz, 2013; Renier et al., 2014; Susaki et al., 2014; Tomer et al., 2014; Yang et al., 2014; Zhang et al., 2014).
Because tissue opacity results mainly from the scattering of light which occurs when the refractive index (RI) of the scatter differs from that of the medium (Helmchen and Denk, 2005), optical clearing methods usually yield tissue transparency via RI matching. A simple strategy is to merely utilize a clearing agent with a high RI approximating to that of fixed tissue $(\sim 1.5)$ (Dodt et al., 2007; Becker et al., 2012; Erturk et al., 2012a,b; Ke et al., 2013; Susaki et al., 2014). The alternative is to intentionally remove hydrophobic lipids which are the main source of light scattering in the fixed brain (Chung et al., 2013; Susaki et al., 2014). Among these techniques, fructose-based SeeDB does not disrupt the plasma membrane, and can therefore clear brain samples labeled with lipophilic dyes that are indispensible for neural tract tracing of post-fixed brains (Ke et al., 2013). However, the viscosity of saturated fructose (e.g., $130 \% \mathrm{wt} / \mathrm{vol}$ at $37^{\circ} \mathrm{C}$ ) is extremely high, which limits its ability to permeate into brain samples as well as making solution preparation and manipulation difficult. Although incubation at a higher temperature could improve these permeability and fluidity issues, this would cause partial quenching of fluorescent proteins (Ke et al., 2013). Arterial perfusion-assisted delivery is also efficient for rapid diffusion of 
clearing agents across the whole brain (Yang et al., 2014), but extremely high viscosity prohibits application of SeeDB via arterial perfusion. In fact, it is difficult for SeeDB to clear larger mammalian brains, although SeeDB does render the adult mouse brain sufficiently transparent through immersion. Nevertheless, in mammals with larger brains, particularly in primates, investigation of the wiring diagram of the brain still relies heavily on tract tracing technologies. Thus, there is a compelling need to develop a clearing method which retains the advantages of SeeDB but overcomes its limitations.

Here we report an optical clearing method that uses a gradient of cocktail solutions, termed FRUIT, which is mainly composed of fructose and urea in variable proportions. FRUIT achieved greater tissue transparency and required a shorter clearing time than either fructose or urea alone. Urea-mediated tissue expansion could be controlled by increasing the concentration of fructose in the initial cocktail solution. Importantly, the presence of urea reduced the need for fructose. As compared with the highest concentration of SeeDB, the dynamic viscosity of the final FRUIT cocktail was lowered by over $97 \%$ so as to render the whole adult rabbit brain transparent via arterial perfusion. Under twophoton microscopy, the fluorescence of both enhanced yellow fluorescent protein (eYFP) and DiI was well preserved in the FRUIT-processed brain. The present study provides a new cocktail recipe which underlies a volume-independent and lipophilic tracer-compatible optical tissue clearing method.

\section{MATERIALS AND METHODS MAKEUP OF THE COCKTAIL}

To develop a clearing method which overcomes the limitations of SeeDB while retaining its advantages, we sought to make a cocktail comprising fructose and other available clearing agents which had been subject to careful verification and demonstrated satisfactory performance using adult mouse brain samples. The candidates were determined based on two principles. First, they needed to be compatible with the fluorescent proteins and tracers commonly used in neuroscience research. For whole-brain imaging, neurons of interest and their processes are usually recognized through the use of fluorescent labels. Thus, organic solvents were excluded due to concern regarding fluorescence quenching (Dodt et al., 2007; Becker et al., 2012; Erturk et al., 2012a,b). Given that high concentrations of fructose solutions are themselves relatively viscous, which makes it difficult to remove bubbles, the second principle required that the candidates were highly watersoluble but less bubble-generative. Accordingly, neither ionic nor non-ionic detergents were taken into consideration because they gave rise to bubbles during solution preparation and manipulation (Chung et al., 2013; Susaki et al., 2014). Based on the above two principles, urea was chosen as the other main ingredient of the cocktail solution, which was termed FRUIT.

To determine whether or not fructose and urea can coexist, we conducted pilot tests to examine the simultaneous dissolution of fructose $(80 \% \mathrm{wt} / \mathrm{vol})$ and urea $(24 \% \mathrm{wt} / \mathrm{vol}$, equal to $4 \mathrm{M}$ ) in water. D-fructose, urea and other reagents were purchased from Aladdin Industrial Corporation (Shanghai, China) or Sinopharm Chemical Reagent Company (Beijing, China). Fructose was first completely dissolved in deionized water at about $65^{\circ} \mathrm{C}$. After cooling to $37^{\circ} \mathrm{C}$, urea was added to a final concentration as required. Notably, the mixture remained stable over 3 days at $37^{\circ} \mathrm{C}$ but not at $65^{\circ} \mathrm{C}$, in terms of both appearance (Figure 1A) and RI. Given that SeeDB demonstrated general superiority over Scale (Ke et al., 2013), we decided to dissolve as much urea as possible into the ascending gradient of fructose solutions used in SeeDB so as to maximize the clearing action of the fructose. For convenience, the concentrations of the FRUIT solutions were determined in terms of their fructose concentration. It was found that fructose solutions to $83 \%$ (wt/vol) were capable of dissolving at least $4 \mathrm{M}$ urea whereas a saturated fructose solution ( $130 \% \mathrm{wt} / \mathrm{vol}$ ) could dissolve virtually no urea (Supplementary Figure 1). We then prepared a gradient of FRUIT solutions containing fructose and urea in variable proportions (Supplementary Table 1). For the purpose of comparison, the gradient of FRUIT solutions was initially designed following the gradient of fructose concentrations used in SeeDB. The urea was maintained at $48 \%$ (wt/vol, equal to $8 \mathrm{M}$ ) if possible or otherwise saturated, as the transitory use of $8 \mathrm{M}$ urea significantly accelerated clearing of the brain without damage to fluorescent proteins (Hama et al., 2011). All FRUIT solutions contained 0.5\% (wt/vol) $\alpha$-thioglycerol. FRUIT solutions at a concentration of $100 \%$ (wt/vol) could be stored at $4^{\circ} \mathrm{C}$ without any crystallization. To control tissue expansion, 20 80\% (wt/vol) FRUIT solutions were also prepared with different concentrations of PBS instead of water as indicated in Supplementary Table 1. The RIs of the solutions were measured at $18^{\circ} \mathrm{C}$ using an Abbe refractometer (INESA Instrument, Shanghai, China).

\section{MEASUREMENT OF SOLUTION TRANSMITTANCE}

The light transmittance of the clearing agents was measured using a spectrophotometer (UV2400, Sunny Optical Technology Group, Shanghai, China). The transmittance of solutions containing clearing agents was initially measured using pure water as the control. However, the transmittance of the solutions significantly exceeded baseline in the band from $930 \mathrm{~nm}$ to $1000 \mathrm{~nm}$ (Figures 2A,B), particularly at about $970 \mathrm{~nm}$, because water itself substantially absorbs light at near-infrared wavelengths (Hale and Querry, 1973). This problem had probably been overlooked previously, as the endpoint of the transmittance curve was set before $950 \mathrm{~nm}$ in earlier studies (see Figure 1A in Hama et al., 2011 and Figure1D in Ke et al., 2013). To correct this bias, the transmittance of solutions was first normalized against air and the blank, respectively, after which the mean of the measured values was taken as the true transmittance.

\section{ANIMALS}

All animals were housed and treated in accordance with institutional guidelines. The experimental procedures and housing conditions were approved by the Animal Experiment Committee of the Beijing Institute of Radiation Medicine. C57BL/6N mice, Thyl-YFP (line $\mathrm{H}$ ) mice in a C57BL/6N background and New Zealand rabbits were used for optical clearing.

\section{OPTICAL CLEARING USING FRUIT}

The different FRUIT protocols used on the adult mouse brain are depicted in Figure 3A. The initially designed FRUIT (20:115) 


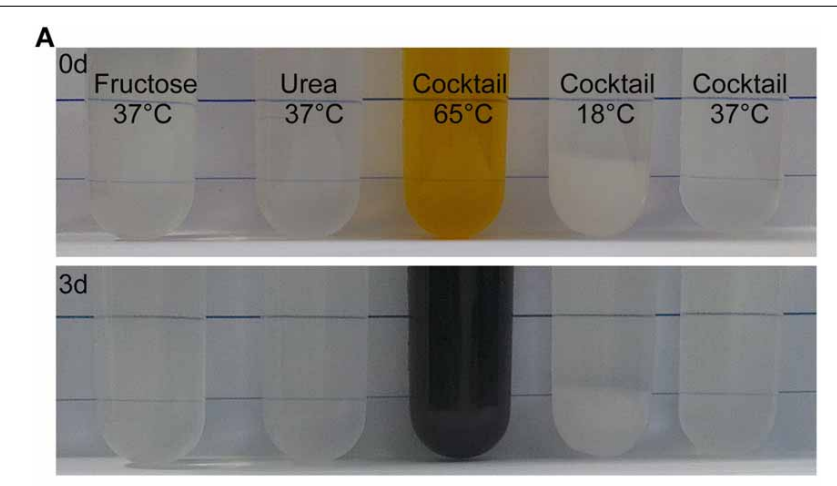

B

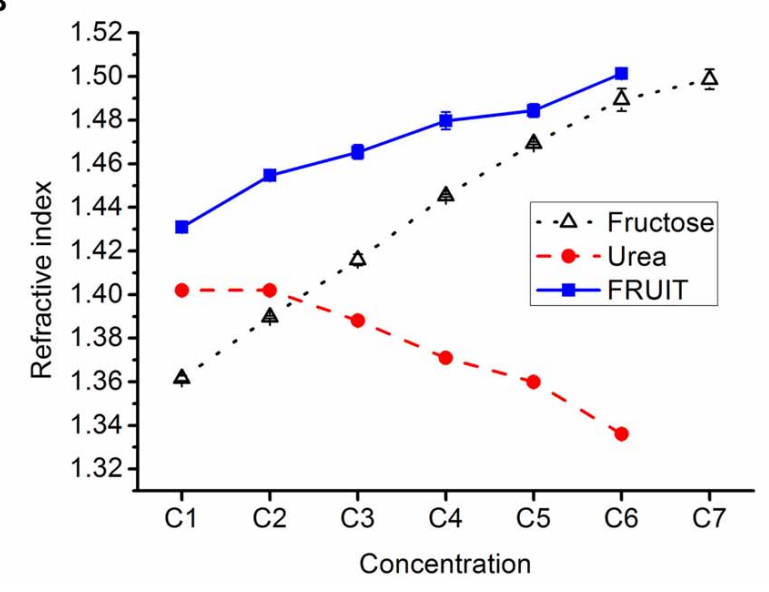

FIGURE 1 | Composition of the cocktail. (A) The fructose solution $(80 \%$ in $\mathrm{wt} / \mathrm{vol})$, urea solution ( $24 \%$ in $\mathrm{wt} / \mathrm{vol}$, equal to $4 \mathrm{M}$ ), and cocktail solution containing $80 \%$ fructose and $24 \%$ urea all remained stable in terms of appearance over 3 days at $37^{\circ} \mathrm{C}$. In contrast, the cocktail solution turned from light brown to dark brown if kept at $65^{\circ} \mathrm{C}$, whereas $8 \mathrm{~g}$ fructose and $2.4 \mathrm{~g}$ urea could not be fully dissolved in $10 \mathrm{ml}$ of aqueous solution at $18^{\circ} \mathrm{C}$. (B) The refractive index (RI) of the different clearing solutions presented as mean $\pm \mathrm{SD}(n=3)$. The composition of these solutions is shown in Supplementary Tables 1-3.

procedure was performed as follows. Adult mice over 70 days of age were deeply anesthetized with an intraperitoneal overdose of sodium pentobarbital $(70 \mathrm{mg} / \mathrm{kg}$ body weight) and transcardially perfused with $1 \times$ PBS followed by $4 \%(\mathrm{wt} / \mathrm{vol})$ paraformaldehyde (PFA) in $1 \times$ PBS. The whole brains were excised and then post-fixed in the same fixative at $4^{\circ} \mathrm{C}$ overnight. The brain samples were serially incubated in $20-30 \mathrm{ml}$ of 20,40 , and $60 \%$ (wt/vol) FRUIT, each for $8 \mathrm{~h}$ in $50-\mathrm{ml}$ conical tubes with gentle rotation $(\sim 4 \mathrm{rpm})$ at $37^{\circ} \mathrm{C}$. The samples were then incubated in $80 \%(\mathrm{wt} / \mathrm{vol})$ FRUIT for $12 \mathrm{~h}, 100 \%$ FRUIT for $12 \mathrm{~h}$ and finally $115 \%$ FRUIT for $24 \mathrm{~h}$ with gentle rotation at $37^{\circ} \mathrm{C}$. In the case of the Thyl-YFP (line $\mathrm{H}$ ) mice, the samples were covered with foil and protected from light during clearing. After noting that the samples showed volume expansion under the above conditions, we considered choosing an appropriate start concentration and elevating the ionic osmotic pressure in the FRUIT solutions to control FRUIT-mediated tissue expansion. In order to achieve maximal transparency without deformation, the optimal FRUIT protocol was established as follows: $8 \mathrm{~h}$ each in 35,40 , and $60 \%$ (wt/vol) FRUIT, $12 \mathrm{~h}$ in $80 \%(\mathrm{wt} / \mathrm{vol}$ ) FRUIT and $24 \mathrm{~h}$ in $100 \%$ $\left(\mathrm{wt} / \mathrm{vol}\right.$ ) FRUIT. Incubation at a temperature over $37^{\circ} \mathrm{C}$ is not recommended due to concern regarding the breakdown of FRUIT. Samples could be stored in 100\% (wt/vol) FRUIT over 2 months at $4^{\circ} \mathrm{C}$.

\section{PERFUSION-ASSISTED CLEARING USING FRUIT}

For perfusion-assisted clearance of the brain, 3 month old adult rabbits were anesthetized with an intravenous injection of sodium pentobarbital ( $35 \mathrm{mg} / \mathrm{kg}$ body weight) through the auricular vein. The rabbit was secured in a supine position and the common carotid arteries and internal jugular veins were exposed. The bilateral common carotid arteries were ligated and the internal jugular veins were opened. A needle was inserted into each common carotid artery at the distal segment to allow for cerebral perfusion of $1 \times$ PBS followed by $4 \%(\mathrm{wt} / \mathrm{vol})$ PFA in $1 \times$ PBS. The rabbit brain was then sequentially perfused with $50 \mathrm{ml}$ of 20,40 , 60,80 , and $100 \%$ (wt/vol) FRUIT solutions through an injection pump (ZT-500A1, Z\&T Medical Treatment, Shenzhen, China) at a flow rate of $5 \sim 10 \mathrm{ml} / \mathrm{h}$ at room temperature. Arterial perfusion of SeeDB solutions could not be completed because $130 \%$ $\left(\mathrm{wt} / \mathrm{vol}\right.$ ) fructose was too viscous to be infused even at $37^{\circ} \mathrm{C}$.

\section{OPTICAL CLEARING WITH SeeDB}

For clearing with SeeDB (Ke et al., 2013), PFA-fixed brain samples excised from the adult mouse were serially incubated in $20-30 \mathrm{ml}$ of 20,40 , and $60 \%$ (wt/vol) fructose, each for $8 \mathrm{~h}$ with gentle rotation at $37^{\circ} \mathrm{C}$. Samples were then incubated in $80 \%(\mathrm{wt} / \mathrm{vol})$ fructose for $12 \mathrm{~h}, 100 \%$ fructose for $12 \mathrm{~h}, 115 \%$ fructose for $24 \mathrm{~h}$ and finally $130 \%$ fructose for $24 \mathrm{~h}$ with gentle rotation at $37^{\circ} \mathrm{C}$. All fructose solutions contained $0.5 \% \alpha$-thioglycerol. Figure 3A schematically depicts the clearing procedure. For Thyl-YFP (line $\mathrm{H})$ mice, the samples were covered with foil and protected from light during clearing.

\section{OPTICAL CLEARING WITH SCALE AND DESCENDING GRADIENT UREA SOLUTIONS}

Given that Scale has been systematically compared with SeeDB (Ke et al., 2013), we only compared the clearing competence between FRUIT and Scale A2 or a descending gradient of urea solutions within a similar incubation period. For clearing with Scale (Hama et al., 2011), PFA-fixed brain samples excised from the adult mouse were transferred to $20-30 \mathrm{ml}$ of Scale A2 solutions containing $4 \mathrm{M}$ urea, $10 \%(\mathrm{wt} / \mathrm{vol})$ glycerol, and $0.1 \%$ (vol/vol) Triton X-100 and incubated at $37^{\circ} \mathrm{C}$ with gentle rotation for 4.5 days. For tissue clearance using a descending gradient of urea solutions, PFA-fixed brain samples excised from the adult mouse were serially incubated in $8 \mathrm{M}$ (equal to $48 \% \mathrm{wt} / \mathrm{vol}$ ) urea for $16 \mathrm{~h}, 6.16 \mathrm{M}(37 \% \mathrm{wt} / \mathrm{vol})$ urea for $8 \mathrm{~h}, 4.3 \mathrm{M}(26 \% \mathrm{wt} / \mathrm{vol})$

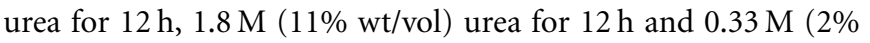
$\mathrm{wt} / \mathrm{vol}$ ) urea for $24 \mathrm{~h}$ with gentle rotation at $37^{\circ} \mathrm{C}$. Figure $3 \mathrm{~A}$ schematically illustrates these clearing procedures.

\section{MEASUREMENT OF TISSUE TRANSMITTANCE}

The light transmittance of the brain samples was measured using a spectrophotometer (UV2400, Sunny Optical Technology Group, Shanghai, China). As the light scattering of the adult brain is non-homogeneous due to the presence of highly myelinated 

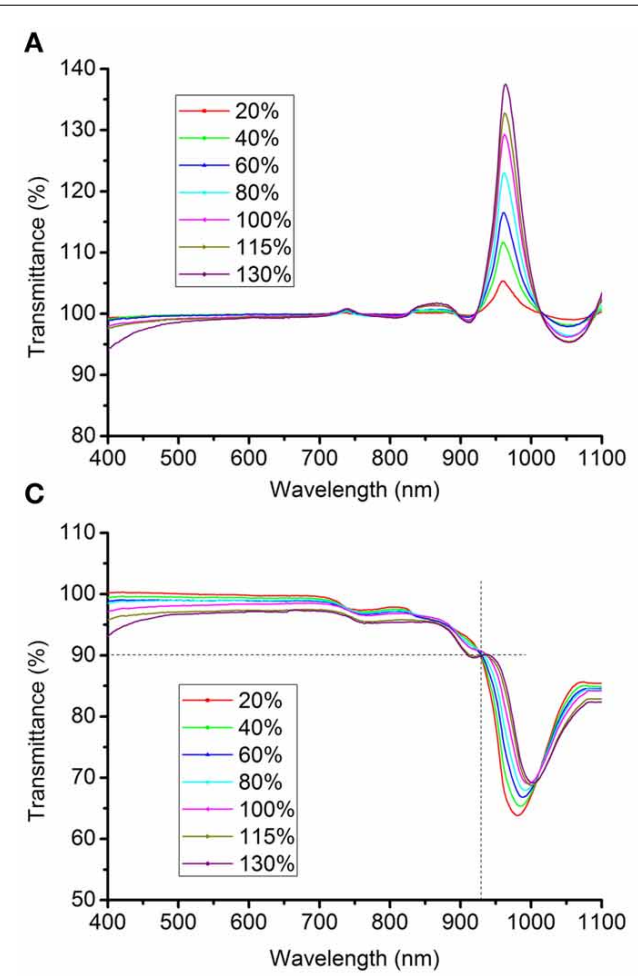

FIGURE 2 | Transmittance curves of clearing solutions. (A,B) The transmittance of SeeDB (A) or FRUIT (B) solutions at different concentrations, normalized against pure water. The transmittance of the solutions exceeded the baseline in the band from 930 to $1000 \mathrm{~nm}$, particularly at about $970 \mathrm{~nm}$,
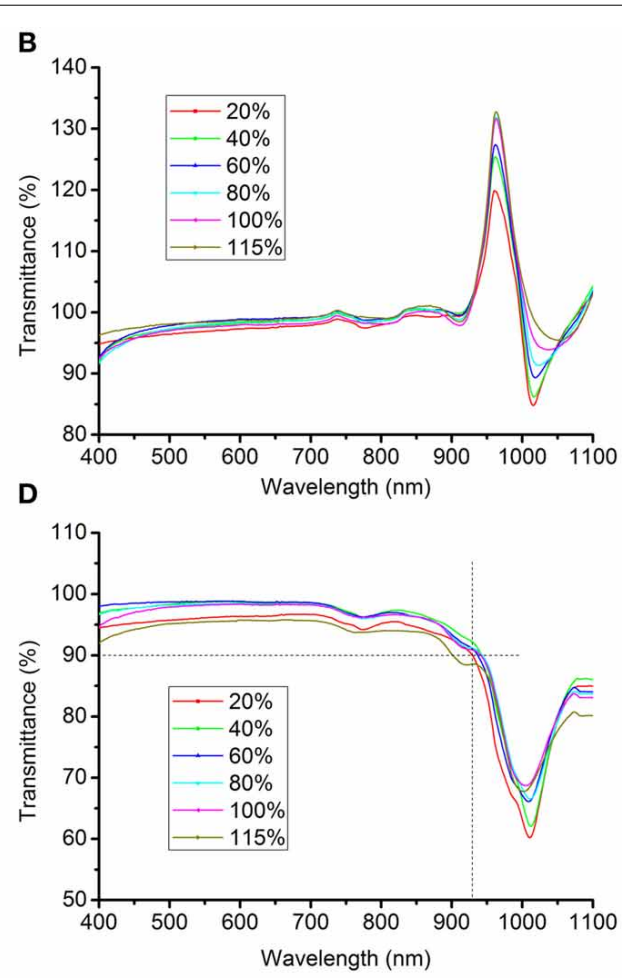

because water itself substantially absorbs light at near-infrared wavelengths (Hale and Querry, 1973). (C,D) The corrected transmittance curves of the SeeDB (C) and FRUIT (D) solutions at different concentrations, normalized against air and the blank (see Materials and Methods). structures (Ke et al., 2013), the medial-to-lateral transmittance of a hemi-brain was measured through its thickest part, with the midplane facing the light. Cleared hemi-brains without liquid can act as a convex lens due to the mismatch in RI between the air and the cleared samples (Susaki et al., 2014). To avoid such a possible convex lens effect, the samples were secured in quartz cuvettes filled with clearing agents with their midplane closely adherent to the sidewall of the cuvette, and their transmittance was normalized against the solutions containing different clearing agents. To ensure strict control, the brains were cut into equal left and right halves along the midline in several experiments, as illustrated in Figures 3G-I.

\section{EXAMINATION OF SAMPLE DEFORMATION}

For the measurement of sample deformation, the brains were cut into left and right halves along the midline, and the hemiforebrains were excised. The samples were then incubated in SeeDB or FRUIT solutions as illustrated in Figure 3A. Photos of samples without liquid were taken before, during and after clearing, with their ventral plane on a glass plate. Based on top view photos, the size of the samples was examined with reference to the grid $(5 \times 5 \mathrm{~mm})$ in the same picture.

\section{ASSESSMENT OF LIGHT SCATTERING}

The brains were cut into equal left and right halves along the midline, and the samples were incubated in SeeDB or FRUIT (35:100). A laser beam at infrared wavelength (1064 $\mathrm{nm}$ was used due to the availability of a laser source) was positioned perpendicular to the midplane of the hemi-brains and the diameter of the laser spot on the midplane was adjusted to $2 \mathrm{~mm}$. The laser intensity through the hemi-brains was measured in a dark room using a laser power meter (PD300, Ophir Photonics, Jerusalem, Israel) at angles of 0,45 and 90 degrees from the incident laser, before and after clearing. The measured values were then normalized to the intensity of the incident laser for comparison. The assessment of light scattering is schematically illustrated in Supplementary Figure 2.

\section{EXAMINATION OF SOLUTION VISCOSITY}

Dynamic viscosity of solutions was measured at room temperature $\left(29^{\circ} \mathrm{C}\right)$ or $37^{\circ} \mathrm{C}$ using a viscometer (DV-S, Brookfield, Middleboro, MA). For the fluidity test, the pipettes were filled with $100 \%$ FRUIT or $130 \%$ (wt/vol) fructose solutions, and then maintained vertically to allow the solutions to fall by gravity at room temperature $\left(18^{\circ} \mathrm{C}\right)$ or $37^{\circ} \mathrm{C}$. The time for the solution with low viscosity to run out was recorded.

\section{Dil LABELING}

PFA-fixed brains were cut into left and right hemi-brains along the midline. As reported in the previous study (Lin et al., 2000), a small incision was made with a scalpel in the cingulate on each side, and a small DiI $\mathrm{C}_{18}$ (3) crystal (Invitrogen, Carlsbad, CA) was placed into the incision. The hemi-brain samples were 


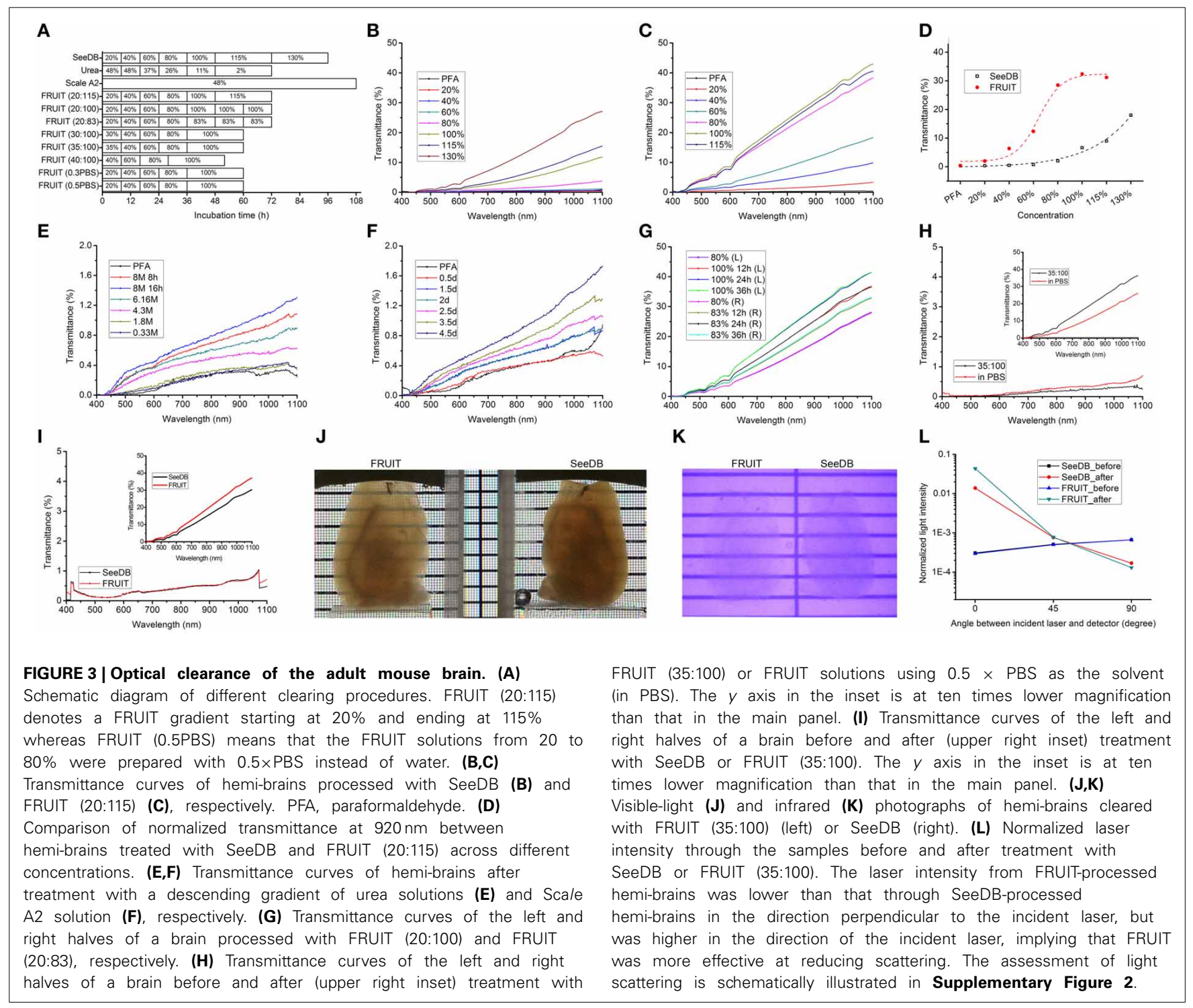

then incubated in $2 \%(\mathrm{wt} / \mathrm{vol}) \mathrm{PFA}$ in PBS at $37^{\circ} \mathrm{C}$ for 14 days, after which they were serially incubated in a gradient of SeeDB or FRUIT (35:100) solutions, respectively. During incubation and clearing, the samples were covered with foil and protected from light. Cleared samples were subjected to two-photon imaging using an upright microscope (FV10MP-BXD4CH, Olympus, Tokyo, Japan) and the images were acquired at $990 \mathrm{~nm}$ excitation.

\section{TWO-PHOTON IMAGING OF CLEARED SAMPLES}

The brain samples from Thy1-YFP (line H) mice were placed in a hand-made chamber filled with clearing agents. For SeeDBtreated samples, $130 \%(\mathrm{wt} / \mathrm{vol}$ ) fructose was not used for immersion due to the fact that its extreme viscosity would cause an uneven RI distribution after evaporation of water during imaging and might impair image quality. Although previously suggested as an immersion agent (Ke et al., 2013), 2, 2'-thiodiethanol was also not used, due to lack of availability. As a result, 100\% FRUIT $(\mathrm{wt} / \mathrm{vol}, R I=1.48)$ or $115 \%$ FRUIT ( $\mathrm{wt} / \mathrm{vol}, R I=1.50)$ was used for immersion for FRUIT (35:100)- or SeeDB-cleared samples. An upright multiphoton microscope (FV10MP-BXD4CH, Olympus) was used for two-photon imaging with $920 \mathrm{~nm}$ excitation. Images at a resolution of $512 \times 512$ pixels were collected with a 25 $\times$ objective (Olympus, $N A=1.0$, working distance $=4.0 \mathrm{~mm}$ ). Image analysis and presentation were performed with FluoView 1000 software (Olympus).

\section{RESULTS}

\section{SYNERGISTICALLY IMPROVED CLEARING COMPETENCE OF THE COCKTAIL}

According to the theory of RI matching, the proximity of the RI to that of fixed tissues $(\sim 1.5)$ predicts good clearing competence of agents (Ke et al., 2013). Our measurement revealed that the RIs of the FRUIT solutions were closer to 1.5 than those of the corresponding fructose or urea solutions (Figure 1B), suggesting that FRUIT may achieve better tissue clearance. 
As tissue transparency was represented by light transmittance of brain samples under immersion in different aqueous solutions containing clearing agents, we first measured the transmittance of various clearing agents to assess their influence on the transmittance of samples. Unlike the RI, the transmittance did not differ significantly across solutions containing different clearing agents (Figures 2C,D), which suggests that light absorption by different solutions at most only slightly biases the transmittance of samples, albeit that this possibility could not be completely excluded.

We next subjected PFA-fixed adult mouse brain samples to different clearing procedures as indicated in Figure 3A. For the purpose of comparison, the gradient of FRUIT solutions was initially designed following the gradient of fructose concentrations used in SeeDB. The transmittance of hemi-brains treated with SeeDB and FRUIT (20:115) shared two common features. First, the transmittance gradually improved as the wavelength increased from 400 to $1100 \mathrm{~nm}$ (Figures 3B,C). Second, the transmittance also improved in a concentration-dependent manner, although at different rates (Figure 3D). Specifically, the transmittance of SeeDB-treated tissues was poor when the fructose concentration was lower than $80 \%$, but improved sharply thereafter. This may explain why the typical SeeDB procedure ended with $130 \%$ fructose, despite the handling difficulty caused by extreme viscosity (Ke et al., 2013). In contrast, FRUIT-processed tissues already displayed considerable transmittance at the initial concentration (i.e., 20\%) and continued to improve until 100\%. These two features provide further assurance that the transmittance of cleared tissues reliably reflects actual tissue transparency.

We then compared the transparency of hemi-brains treated with different clearing agents. As predicted from the RIs, a gradient of FRUIT solutions achieved better tissue clearance than SeeDB (Figure 3D). However, both the Scale A2 solution and a descending gradient of urea solutions produced only negligible transmittance within a similar incubation period (Figures 3E, F), suggesting that the combination of fructose and urea exerted a synergistic rather than an additive effect on clearance competence.

We found that $115 \%$ FRUIT treatment did not provide any additional gain in tissue transparency (Figures 3C, D). To determine a suitable final concentration for the gradient, we then compared tissue transparency between 100\% FRUIT and 83\% FRUIT in which the concentration of urea was retained at $4 \mathrm{M}$ (Supplementary Figure 1 and Supplementary Table 1). After $80 \%$ FRUIT, the former resulted in better tissue transparency (Figure 3G). Moreover, the best tissue clearance by the $100 \%$ FRUIT solution was achieved after $24 \mathrm{~h}$ and maintained thereafter (Figure 3G), suggesting that a $24 \mathrm{~h}$ incubation was sufficient for $100 \%$ FRUIT to achieve optimal clearance of PFA-fixed adult mouse hemi-brains.

\section{CONTROLLABLE TISSUE DEFORMATION BY THE COCKTAIL}

Tissue deformation commonly occurs during the process of optical clearing (Dodt et al., 2007; Hama et al., 2011; Erturk et al., 2012a; Chung et al., 2013; Ke et al., 2013). We noted that the final volume of brain samples also increased after treatment with FRUIT (20:115) (Figure 4A). Given that tissue expansion alone can reduce light scattering (Ke et al., 2013), there was a potential concern that the better tissue transparency afforded by FRUIT might be due to tissue expansion. We therefore, examined this possibility, and found that the brain stopped expanding at a FRUIT concentration of $40 \%$, after which it partially recovered (Figure 4A). In contrast, the transparency of the same hemibrain was continuously enhanced up to a concentration of $100 \%$ (Figures 3C, D). Therefore, it seemed unlikely that the better tissue transparency observed with FRUIT treatment was mainly mediated by tissue expansion. This concern was later completely resolved when FRUIT-caused tissue expansion was effectively controlled.

By comparing FRUIT with SeeDB and Scale, we concluded that FRUIT-mediated tissue expansion was mainly due to the high concentration of urea. Although reducing the level of urea in FRUIT solutions was beneficial for deformation control, this option was discarded because it would be detrimental to tissue transparency. As fructose can prevent tissue expansion through dehydration in a concentration-dependent manner (Ke et al., 2013; Susaki et al., 2014), one expansion-controlling strategy was to increase fructose in the starting concentration of the gradient of FRUIT solutions. We also found that the final size of the brain was seemingly equal to the size after $8 \mathrm{~h}$ incubation in the initial concentration of FRUIT, i.e., 20\%, regardless of the dynamic volume change across the gradient of FRUIT solutions (Figure 4A). This finding inspired us to consider that choosing an appropriate starting concentration was enough to control FRUIT-mediated tissue expansion. We therefore, compared brain size after brief treatment in various concentrations of FRUIT solution. It was found that only 35\% FRUIT was able to preserve the original size of the brain, whereas a lower or higher concentration caused expansion or shrinkage, respectively (Figure 4B). Notably, onestep treatment with $100 \%$ FRUIT caused severe shrinkage of the samples (Figure 4B). Based on these results, we modified the initial FRUIT concentration of the gradient. As expected, a gradient of FRUIT solutions starting from 35\% preserved the brain size well, whereas those with a lower or higher start concentration deformed the brain (Figure 4C).

Brain deformation also reflects an imbalance of ionic osmotic pressure between the inside and outside of the brain. Thus, another expansion-controlling strategy we considered was to elevate the ionic osmotic pressure in the FRUIT solutions. Consequently, 20-80\% FRUIT solutions were prepared with different concentrations of PBS instead of water (Supplementary Table 1). This revealed that $0.5 \times$ PBS in $20-80 \%$ FRUIT solutions was sufficient to control for expansion of the brain (Figure 4D).

In order to achieve maximal transparency without deformation, we further compared brain transparency between the two simple but effective strategies for expansion control. After finding that the FRUIT procedure beginning at a concentration of $35 \%$ rendered the brain clearer than that using $0.5 \times \mathrm{PBS}$ as a solvent (Figure 3H), a gradient comprising 35, 40, 60, 80, and $100 \%$ FRUIT solutions (see Figure 3A), was finally selected as the optimal approach. However, it should be noted that the optimal procedure might vary depending on animal age, tissue volume, fixation time, solution temperature and other factors. 


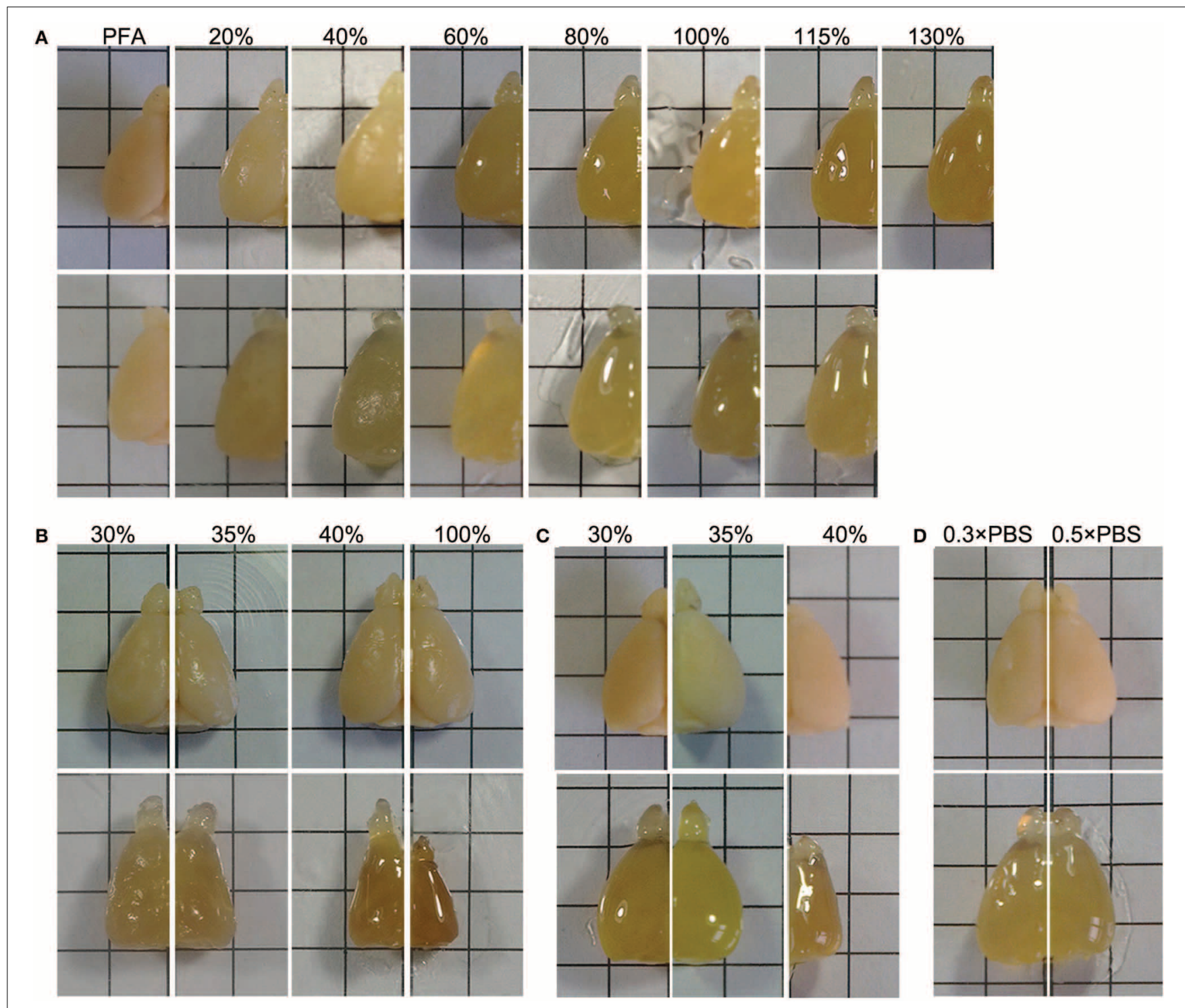

FIGURE 4 | Control of tissue deformation. (A) The hemi-brains were sequentially treated with a gradient of SeeDB (upper row) or FRUIT (20:115) (lower row) solutions at $37^{\circ} \mathrm{C}$. The final volumes of the brain samples show expansion after treatment with the FRUIT (20:115) but not the SeeDB protocol. For FRUIT-processed samples, the final size is seemingly equal to the size after $8 \mathrm{~h}$ incubation in the initial concentration of FRUIT, i.e., $20 \%$ FRUIT, regardless of the dynamic volume change across the gradient of FRUIT solutions. PFA, paraformaldehyde. (B) The hemi-brains before (upper row) and after (lower row) the $8 \mathrm{~h}$ incubation in single concentrations of FRUIT solutions at $37^{\circ} \mathrm{C}$. Only $35 \%$ FRUIT is able to preserve the original size of the brain, whereas a lower or higher concentration causes expansion or shrinkage. (C) The hemi-brains before (upper row) and after (lower row) sequential treatment with the gradient of FRUIT solutions at $37^{\circ} \mathrm{C}$ starting from different concentrations. The gradient of FRUIT solutions starting from $35 \%$ preserved brain size well, whereas those with a lower or higher start concentration deformed the brain. (D) The hemi-brains before (upper row) and after (lower row) treatment with FRUIT solutions using $0.3 \times$ PBS or $0.5 \times$ PBS as the solvent at $37^{\circ} \mathrm{C}$. $0.5 \times$ PBS in $20-80 \%$ FRUIT solutions is sufficient to control expansion of the brain. This figure was set to demonstrate the changes of brain sizes in air in the epi-illumination mode (up-down). Brain transparency would be significantly improved by photographing the samples in solutions in the trans-illumination mode (down-up), as shown in Figures 3J, K. Grid size: $5 \times 5 \mathrm{~mm}$.

\section{SCALABLE CLEARANCE WITH eYFP AND Dil COMPATIBILITY}

When tissue expansion was controlled, FRUIT (35:100) still achieved better brain transparency than SeeDB across the full spectrum from $400 \mathrm{~nm}$ to $1100 \mathrm{~nm}$ (Figure 3I), suggesting that FRUIT-mediated transparency occurred independent of brain expansion. Notably, the better tissue transparency afforded by FRUIT was more pronounced when examined under the infrared spectrum vs. visible light (Figures 3J, K), which facilitates twophoton imaging of the whole brain, particularly deep structures.

To explore a possible mechanism accounting for the greater transparency afforded by FRUIT, we assessed the light scattering of cleared brain samples, as the opacity of mammalian tissue results mainly from light scattering rather than light absorption (Ke et al., 2013). The laser intensity from FRUIT-processed 
hemi-brains was lower than that through SeeDB-processed hemibrains in the direction perpendicular to the incident laser but higher in the direction of the incident laser (Figure 3L), meaning that FRUIT was more effective at reducing scattering.

In addition to a synergistic action on tissue transparency, the combination of fructose and urea also resulted in other reciprocally beneficial consequences. Notably, the presence of urea reduced the amount of fructose required. Dynamic viscosity measurements revealed that whether at room temperature (i.e., $29^{\circ} \mathrm{C}$ ) or under mild heating conditions (i.e., $37^{\circ} \mathrm{C}$ ), $100 \%$ FRUIT was 30 times less viscous than a 130\% fructose solution (Figure 5A). Low viscosity not only improved permeability but also accelerated clearance as evidenced by the fact that the total time required for FRUIT (35:100) to clear adult mouse brains was as short as 2.5 days, a reduction of approximately $40 \%$ as compared with SeeDB (Figure 3A). In addition, low viscosity also led to high fluidity (Figure 5B), which made it easy to prepare and exchange the FRUIT solutions as well as to handle samples immersed in them. More importantly, high fluidity made it possible to implement rapid diffusion of clearing agents across the whole brain via arterial perfusion. The gradient of FRUIT solutions, including the $100 \%$ concentration, could be smoothly infused into the common carotid artery so as to render the whole adult rabbit brain transparent (Figures 5C, D), whereas the highest concentration of SeeDB (130\% fructose) was too viscous to be arterially perfused, suggesting that FRUIT could overcome the volume limitation on the brain imposed by SeeDB.

Given that urea itself leads to partial denaturation of proteins (Ke et al., 2013), a possible concern was that FRUIT might also cause damage to biological samples. However, it seemed that fructose rendered the brain resistant to denaturation by urea. Although Scale-treated samples are very fragile (Hama et al., 2011), FRUIT-treated samples did not display noticeable fragility.

To determine whether or not FRUIT was compatible with the use of representative fluorescent proteins and lipophilic dyes, we examined FRUIT-cleared brains carrying these labels under twophoton microscopy. The fluorescence of cytosolic eYFP in the brains of Thyl-eYFP (line H) transgenic mice was well preserved after FRUIT treatment, similar to the result obtained after SeeDB treatment (Figures 6A-D). Given that eYFP has been reported to be the most susceptible fluorescent protein (Erturk et al., 2012a; Susaki et al., 2014), we speculated that FRUIT would also be valuable for the study of transgenic mice expressing other fluorescent proteins. Interestingly, and also similar to SeeDB (Ke et al., 2013), FRUIT could also clear brains labeled with the lipophilic dye DiI (Figures 6E,F), implying that the plasma membrane of FRUITprocessed brains remains intact and that FRUIT is suitable for tract tracing of post-fixed whole brains.

\section{DISCUSSION}

It was once considered impossible to extract cellular resolution information from unsectioned whole mammalian brains. Recently, tissue optical clearing methods have opened a new avenue to overcome this obstacle (Dodt et al., 2007; Hama et al., 2011; Becker et al., 2012; Erturk et al., 2012a; Chung et al., 2013; Ke et al., 2013; Kim et al., 2013; Kuwajima et al., 2013; Yushchenko and Schultz, 2013; Susaki et al., 2014; Tomer et al., 2014; Yang
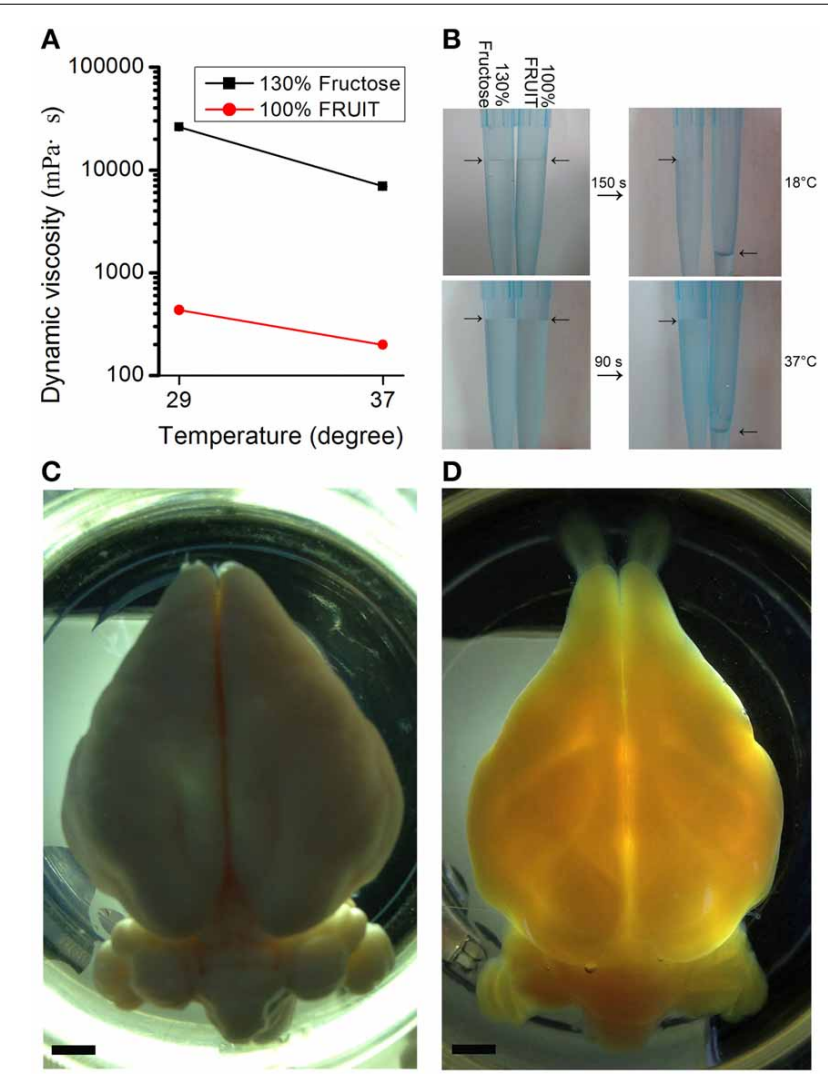

FIGURE 5 | Viscosity-dependent arterial perfusion of clearing agents. (A) The dynamic viscosity of different clearing solutions presented as mean $\pm \mathrm{SD}(n=3) .100 \%$ FRUIT was 30 times less viscous than $130 \%$ fructose solution. (B) Fluidity test of the clearing solutions. 100\% FRUIT ran out through the pipette within $150 \mathrm{~s}$ at $18^{\circ} \mathrm{C}$ (upper row) and within $90 \mathrm{~s}$ at $37^{\circ} \mathrm{C}$ (lower row) whereas $130 \%$ fructose hardly fell. (C,D) Adult rabbit brains after arterial perfusion of paraformaldehyde (C) or the gradient of FRUIT solutions (D). Perfusion of FRUIT solutions rendered the whole brain transparent whereas $130 \%$ SeeDB was too viscous to be arterially perfused. An adult rabbit brain is about 20 times larger in volume than an adult mouse brain. Bar $=5 \mathrm{~mm}$.

et al., 2014; Zhang et al., 2014). Previous clearing methods usually relied on a single clearing agent and rarely obtained a satisfactory balance between tissue transparency and other key parameters such as clearing time, deformation control, fluorescence preservation and operating simplicity (Kim et al., 2013; Yushchenko and Schultz, 2013). Theoretically, it is a promising strategy to make a cocktail of available clearing agents to achieve improved clearing performance without undesirable limitations. In fact, a cocktail strategy has been taken into consideration to direct the design of new clearing methods, such as ClearT (Kuwajima et al., 2013)and CUBIC (Susaki et al., 2014). Although a proposed cocktail does not necessarily translate into a better overall performance, our study provides a paradigm for evidence-based establishment of a new cocktail recipe that offers significantly improved outcomes over those obtained with the individual main ingredients alone. As the number of available clearing agents continues to grow, we believe that a cocktail strategy will prevail in order to ensure various desirable goals in whole-brain imaging studies. 

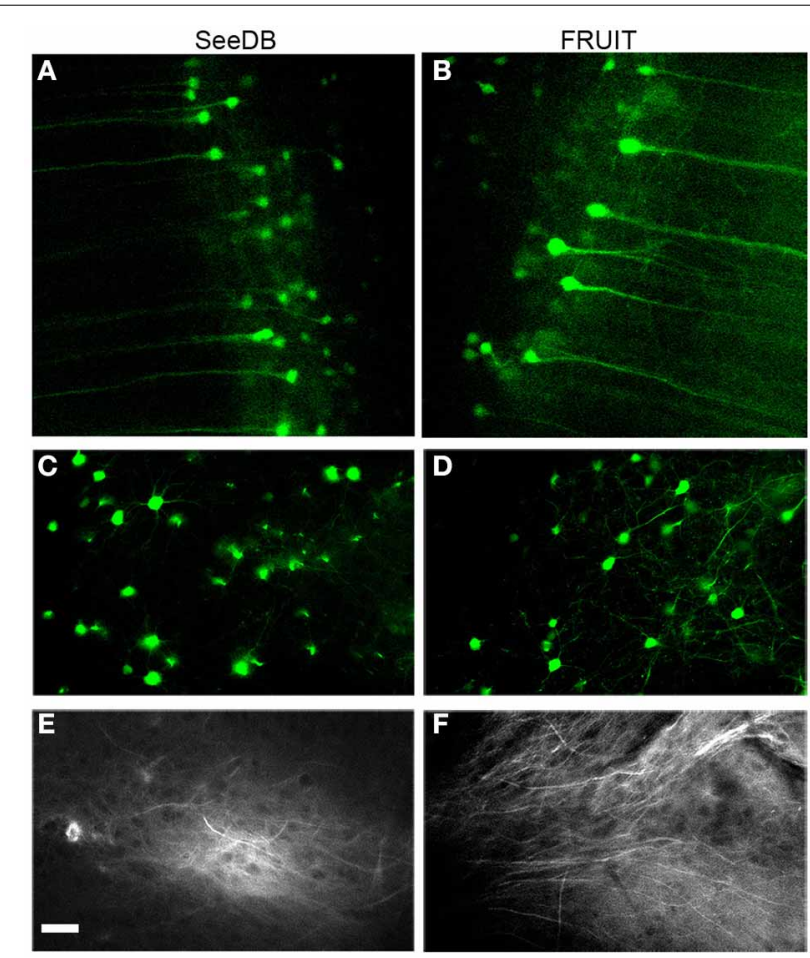

FIGURE 6 | Compatibility with fluorescent proteins or lipophilic tracers. (A, B) Two-photon imaging of cortical pyramidal neurons in Thy1-eYFP (line H) mouse brains cleared with SeeDB (A) or FRUIT (20:115) (B). The fluorescence of cytosolic eYFP was well preserved after treatment with FRUIT (20:115), but the neurons appeared to swell, which is in accordance with the gross appearance of the FRUIT (20:115)-treated brains (see Figure 4A). (C,D) Two-photon imaging of striatal neurons in Thy1-eYFP (line H) mouse brains cleared with SeeDB (C) or FRUIT (35:100), which effectively controlled the brain expansion (D). (E,F) Two-photon imaging of Dil-labeled mouse brains after treatment with SeeDB (E) or FRUIT (35:100) which cause no deformation (F). Like SeeDB, FRUIT preserved the fluorescence of fluorescent proteins or lipophilic tracers. Bar $=50 \mu \mathrm{m}$.

According to predetermined principles regarding good fluorescence compatibility and effective bubble control, we chose urea as the other main ingredient for our FRUIT solutions. In theory, there are two different design strategies to create a cocktail of fructose and urea. One is to add increasing amounts of fructose into a constant concentration of urea solution, and the other is to dissolve as much urea as possible into an ascending gradient of fructose solutions. Given that SeeDB demonstrates general superiority over Scale (Ke et al., 2013), we adopted the latter strategy so as to maximize the clearing actions of fructose. Our choice is further justified by the following two points. First, keeping urea at a constant concentration (e.g., $4 \mathrm{M}$ ) would impose restrictions on the action of urea in FRUIT solutions at lower concentrations, as FRUIT to a concentration of $80 \%$ achieved considerable tissue transparency mainly through the use of urea at high concentrations above $4 \mathrm{M}$. Second, keeping urea constantly at $4 \mathrm{M}$ would also reduce the solubility of fructose in FRUIT solutions at higher concentrations, whereas $100 \%$ FRUIT yielded greater brain transparency than $83 \%$ FRUIT.
Although the success of urea-based Scale is generally below that of fructose-based SeeDB (Ke et al., 2013), our study clearly demonstrates that urea plays a unique and important role in the applicability of FRUIT. Impressively, urea synergistically enhanced the clearing competence of fructose. We also noted two facts regarding tissue clearance by FRUIT. Firstly, to a concentration of $80 \%$, FRUIT achieved much better tissue clearance than SeeDB. Secondly, treatment with $115 \%$ FRUIT (containing $0.33 \mathrm{M}$ urea) following $100 \%$ FRUIT did not improve tissue transparency. These two facts suggest that urea may only exert an enhancing effect at certain concentrations. The generality and underlying mechanisms of this effect remain to be clarified.

The combination of fructose and urea also results in other reciprocally beneficial consequences. Among these, low viscosity is the most valuable achievement of FRUIT over SeeDB. Low viscosity improves permeability and accelerates clearance as evidenced by the reduced total time required for FRUIT, as compared to SeeDB, to clear adult mouse brains, in both cases under immersion conditions. Low viscosity also translates into high fluidity, which is a prerequisite for rapid diffusion of clearing agents across the whole brain via arterial perfusion. Remarkably, perfusion of a gradient of FRUIT solutions results in transparency of the whole adult rabbit brain which is about 20 times larger than its mouse counterpart and prohibitive for SeeDB. As perfusion-assisted release of clearing agents is not restricted by organ volume (Yang et al., 2014), we envision that FRUIT could even be applicable to the optical clearing of primate brains.

Although urea is reported to cause partial protein denaturation (Hama et al., 2011), our observations argue that this may not be true for urea-containing FRUIT. Firstly, unlike Scale-treated samples (Hama et al., 2011), FRUIT-treated samples were not noticeably fragile. Secondly, but more importantly, FRUIT was shown to be compatible with the lipophilic dye DiI, which implies that the plasma membrane of FRUIT-processed brains remains intact (Ke et al., 2013). These facts suggest that the fructose in the FRUIT solutions may be protective, helping to preserve the plasma membrane in the tissue. This feature distinguishes FRUIT as well as SeeDB from other optical clearing methods which are not suitable for tract tracing of post-fixed whole brains. However, SeeDB and our method would not facilitate immunostaining of the whole brain (Chung et al., 2013; Renier et al., 2014; Susaki et al., 2014; Tainaka et al., 2014; Yang et al., 2014), because they are not intentionally designed to remove lipids which render tissue poorly accessible to molecular probes, such as antibodies.

By means of arterial perfusion, FRUIT can achieve tissue clearance independent of brain volume limitations. Although CUBIC (Susaki et al., 2014) and a modified version of CLARITY (Yang et al., 2014) are also scalable clearing methods, both of them render the whole mammalian brain transparent by removing hydrophobic lipids, which makes it impossible to clear DiIlabeled brain samples. Thus, FRUIT represents a distinctive clearing method which is both independent of brain volume and compatible with lipophilic tracers. In the present study, we only conducted systemic comparisons between our method and SeeDB, in terms of clearing performance, deformation control, DiI compatibility and solution viscosity. Extensive comparisons across existing methods have been performed in previous literatures (see 
Table 1 in Kim et al., 2013, Table 1 in Yushchenko and Schultz, 2013, Supplementary Table 4 in Ke et al., 2013 and Table S1 in Yang et al., 2014). These comparisons suggest that no single clearing technique can satisfy the many goals of whole-brain imaging studies. Researchers may choose the most appropriate one out of the available clearing techniques or even adopt simultaneous or sequential use of them, depending on brain volume, labeling method, and observation tool.

It should also be kept in mind that the same clearing methods would perform differently under different experimental settings. Under confocal microscopy, SeeDB only marginally improved the imaging depth of cultured neurospheres that were $100 \mu \mathrm{m}$ in diameter (Boutin and Hoffman-Kim, 2014), whereas the imaging depth of the adult mouse brain could reach almost $6000 \mu \mathrm{m}$ after treatment with SeeDB under optimal two-photon microscopy (Ke et al., 2013). This example suggests that even the same clearing method may afford different imaging depths under different forms of microscopy.

Rapid development of whole-brain clearing methods also creates unprecedented demands for high-performance imaging platforms (Dodt et al., 2007; Hama et al., 2011; Becker et al., 2012; Erturk et al., 2012a,b; Chung et al., 2013; Ke et al., 2013; Kuwajima et al., 2013; Susaki et al., 2014; Yang et al., 2014). In order to achieve whole-brain imaging at a cellular resolution, it is necessary to utilize long working distance, high numerical aperture, and multi-immersion objectives (Yang et al., 2014). For samples as large as rabbit brains, it remains challenging to obtain whole-brain imaging using available two-photon microscopes. Although two-photon microscopy works on FRUIT-processed mouse brain samples, light-sheet illumination (Truong et al., 2011) could be integrated to significantly accelerate the image acquisition process.

\section{ACKNOWLEDGMENTS}

The authors thank Guo-Shi Bao for insightful discussion, Qin$\mathrm{Zhi} \mathrm{Xu}$ and Ping-Kun Zhou for the use of the laboratory, and Rowan Tweedale for editing the manuscript. This work was supported by the National Key Basic Research and Development Program (973), Grant Nos. 2011CB707800 and 2011CB809102, the Strategic Priority Research Program of the Chinese Academy of Sciences, Grant Nos. XDB02030300 and XDB02050400, and the Natural Science Foundation of China, Grant Nos. 91132301, $91432302,31222025,31171025$, and 61275194.

\section{SUPPLEMENTARY MATERIAL}

The Supplementary Material for this article can be found online at: http://www.frontiersin.org/journal/10.3389/fnana.2015.

\section{9/abstract}

\section{Supplementary Figure 1 | Solubility of urea in a gradient of fructose} solutions at $37^{\circ} \mathbf{C}$. Both fructose and urea are highly water soluble, and the solubility of urea decreases in converse with the fructose concentration. Fructose solutions to $83 \%$ (wt/vol) are able to dissolve at least $4 \mathrm{M}$ urea, whereas saturated fructose solution can dissolve virtually no urea.

\section{Supplementary Figure 2 | Schematic diagram of light scattering} assessment. A laser beam was positioned perpendicular to the midplane of the hemi-brains and the laser intensity through the sample was measured using a laser power meter placed at angles of 90 (D1), 45 (D2), and 0 degrees (D3) from the incident laser. $M$ : the place of a monitor

S: the sample.

\section{REFERENCES}

Becker, K., Jahrling, N., Saghafi, S., Weiler, R., and Dodt, H. U. (2012). Chemical clearing and dehydration of GFP expressing mouse brains. PLoS ONE 7:e33916. doi: 10.1371/journal.pone.0033916

Boutin, M. E., and Hoffman-Kim, D. (2014). Application and assessment of optical clearing methods for imaging of tissue-engineered neural stem cell spheres. Tissue Eng. Part $C$ Methods. doi: 10.1089/ten.tec.2014.0296. [Epub ahead of print]. Available online at: http://online.liebertpub.com/doi/abs/10.1089/ten.TEC.2014.0296?url_ver=Z39. 88-2003\&rfr_id=ori\%3Arid\%3Acrossref.org\&rfr_dat=cr_pub\%3Dpubmed

Chung, K., Wallace, J., Kim, S. Y., Kalyanasundaram, S., Andalman, A. S., Davidson, T. J., et al. (2013). Structural and molecular interrogation of intact biological systems. Nature 497, 332-337. doi: 10.1038/nature12107

DeFelipe, J. (2010). From the connectome to the synaptome: an epic love story. Science 330, 1198-1201. doi: 10.1126/science.1193378

Dodt, H. U., Leischner, U., Schierloh, A., Jahrling, N., Mauch, C. P., Deininger, K., et al. (2007). Ultramicroscopy: three-dimensional visualization of neuronal networks in the whole mouse brain. Nat. Methods 4, 331-336. doi: 10.1038/nmeth1036

Erturk, A., Becker, K., Jahrling, N., Mauch, C. P., Hojer, C. D., Egen, J. G., et al. (2012a). Three-dimensional imaging of solvent-cleared organs using 3DISCO. Nat. Protoc. 7, 1983-1995. doi: 10.1038/nprot.2012.119

Erturk, A., Mauch, C. P., Hellal, F., Forstner, F., Keck, T., Becker, K., et al. (2012b). Three-dimensional imaging of the unsectioned adult spinal cord to assess axon regeneration and glial responses after injury. Nat. Med. 18, 166-171. doi: 10.1038/nm.2600

Hale, G. M., and Querry, M. R. (1973). Optical constants of water in the 200-nm to 200-microm Wavelength Region. Appl. Opt. 12, 555-563. doi: 10.1364/AO.12.000555

Hama, H., Kurokawa, H., Kawano, H., Ando, R., Shimogori, T., Noda, H., et al. (2011). Scale: a chemical approach for fluorescence imaging and reconstruction of transparent mouse brain. Nat. Neurosci. 14, 1481-1488. doi: 10.1038/nn.2928

Helmchen, F., and Denk, W. (2005). Deep tissue two-photon microscopy. Nat. Methods 2, 932-940. doi: 10.1038/nmeth818

Ke, M. T., Fujimoto, S., and Imai, T. (2013). SeeDB: a simple and morphologypreserving optical clearing agent for neuronal circuit reconstruction. Nat. Neurosci. 16, 1154-1161. doi: 10.1038/nn.3447

Kim, S. Y., Chung, K., and Deisseroth, K. (2013). Light microscopy mapping of connections in the intact brain. Trends Cogn. Sci. 17, 596-599. doi: 10.1016/j.tics.2013.10.005

Kuwajima, T., Sitko, A. A., Bhansali, P., Jurgens, C., Guido, W., and Mason, C. (2013). ClearT: a detergent- and solvent-free clearing method for neuronal and non-neuronal tissue. Development 140, 1364-1368. doi: 10.1242/dev. 091844

Li, A., Gong, H., Zhang, B., Wang, Q., Yan, C., Wu, J., et al. (2010). Micro-optical sectioning tomography to obtain a high-resolution atlas of the mouse brain. Science 330, 1404-1408. doi: 10.1126/science.1191776

Lin, D. M., Wang, F., Lowe, G., Gold, G. H., Axel, R., Ngai, J., et al. (2000). Formation of precise connections in the olfactory bulb occurs in the absence of odorant-evoked neuronal activity. Neuron 26, 69-80. doi: 10.1016/S08966273(00)81139-3

Oh, S. W., Harris, J. A., Ng, L., Winslow, B., Cain, N., Mihalas, S., et al. (2014). A mesoscale connectome of the mouse brain. Nature 508, 207-214. doi: 10.1038 /nature13186

Renier, N., Wu, Z., Simon, D. J., Yang, J., Ariel, P., and Tessier-Lavigne, M. (2014). iDISCO: a simple, rapid method to immunolabel large tissue samples for volume imaging. Cell 159, 896-910. doi: 10.1016/j.cell.2014.10.010

Susaki, E. A., Tainaka, K., Perrin, D., Kishino, F., Tawara, T., Watanabe, T. M., et al. (2014). Whole-brain imaging with single-cell resolution using chemical cocktails and computational analysis. Cell 157, 726-739. doi: 10.1016/j.cell.2014.03.042

Tainaka, K., Kubota, S. I., Suyama, T. Q., Susaki, E. A., Perrin, D., Ukai-Tadenuma, M., et al. (2014). Whole-body imaging with single-cell resolution by tissue decolorization. Cell 159, 911-924. doi: 10.1016/j.cell.2014.10.034 
Tomer, R., Ye, L., Hsueh, B., and Deisseroth, K. (2014). Advanced CLARITY for rapid and high-resolution imaging of intact tissues. Nat Protoc 9, 1682-1697. doi: 10.1038/nprot.2014.123

Truong, T. V., Supatto, W., Koos, D. S., Choi, J. M., and Fraser, S. E. (2011). Deep and fast live imaging with two-photon scanned light-sheet microscopy. Nat. Methods 8, 757-760. doi: 10.1038/nmeth.1652

Yang, B., Treweek, J. B., Kulkarni, R. P., Deverman, B. E., Chen, C. K., Lubeck, E., et al. (2014). Single-Cell phenotyping within transparent intact tissue through whole-body clearing. Cell 158, 945-958. doi: 10.1016/j.cell.2014.07.017

Yushchenko, D. A., and Schultz, C. (2013). Tissue clearing for optical anatomy. Angew. Chem. Int. Ed. Engl. 52, 10949-10951. doi: 10.1002/anie.201306039

Zhang, M. D., Tortoriello, G., Hsueh, B., Tomer, R., Ye, L., Mitsios, N., et al. (2014). Neuronal calcium-binding proteins $1 / 2$ localize to dorsal root ganglia and excitatory spinal neurons and are regulated by nerve injury. Proc. Natl. Acad. Sci. U.S.A. 111, E1149-E1158. doi: 10.1073/pnas.1402318111

Conflict of Interest Statement: Bing Hou, Dan Zhang and Tianzi Jiang hold a patent (pending) for the FRUIT technique. The authors declare that the research was conducted in the absence of any commercial or financial relationships that could be construed as a potential conflict of interest.

Received: 02 December 2014; paper pending published: 23 December 2014; accepted: 07 February 2015; published online: 24 February 2015.

Citation: Hou B, Zhang D, Zhao S, Wei M, Yang Z, Wang S, Wang J, Zhang X, Liu B, Fan L, Li Y, Qiu Z, Zhang C and Jiang T (2015) Scalable and DiI-compatible optical clearance of the mammalian brain. Front. Neuroanat. 9:19. doi: 10.3389/fnana. 2015.00019

This article was submitted to the journal Frontiers in Neuroanatomy.

Copyright (C) 2015 Hou, Zhang, Zhao, Wei, Yang, Wang, Wang, Zhang, Liu, Fan, Li, Qiu, Zhang and Jiang. This is an open-access article distributed under the terms of the Creative Commons Attribution License (CCBY). The use, distribution or reproduction in other forums is permitted, provided the original author(s) or licensor are credited and that the original publication in this journal is cited, in accordance with accepted academic practice. No use, distribution or reproduction is permitted which does not comply with these terms. 